\title{
SPECTRALLY ARBITRARY TREE SIGN PATTERNS OF ORDER 4*
}

\author{
MARINA ARAV ${ }^{\dagger}$, FRANK HALL $^{\dagger}$, ZHONGSHAN LI $^{\dagger}$, KRISHNA KAPHLE ${ }^{\ddagger}$, AND \\ NILAY MANZAGOL ${ }^{\dagger}$
}

\begin{abstract}
A sign pattern matrix (or a sign pattern, or a pattern) is a matrix whose entries are from the set $\{+,-, 0\}$. An $n \times n$ sign pattern matrix is a spectrally arbitrary pattern (SAP) if for every monic real polynomial $p(x)$ of degree $n$, there exists a real matrix $B$ whose entries agree in sign with $A$ such that the characteristic polynomial of $B$ is $p(x)$. An $n \times n$ sign pattern $A$ is an inertially arbitrary pattern (IAP) if $(r, s, t)$ belongs to the inertia set of $A$ for every nonnegative integer triple $(r, s, t)$ with $r+s+t=n$. Tree sign patterns are investigated, with a special emphasis on $4 \times 4$ tridiagonal sign patterns. The set of spectrally arbitrary sign patterns is a subset of the set of potentially stable sign patterns, and for tree sign patterns of order 4 , the set of all potentially stable sign patterns is known. The main results are obtained by determining which of these potentially stable sign patterns are SAPs. Necessary and sufficient conditions for an irreducible $4 \times 4$ tridiagonal pattern to be an SAP are found. As a result, all $4 \times 4$ tree sign patterns that are SAPs are characterized. A new technique, an innovative application of Gröbner bases for demonstrating that a sign pattern is not potentially nilpotent, is introduced. Connections between the SAP classes and the classes of potentially nilpotent and potentially stable patterns are explored. Some interesting open questions are also provided.
\end{abstract}

Key words. Sign pattern matrix, Spectrally arbitrary pattern, Inertially arbitrary pattern, Tree sign pattern, Potentially nilpotent pattern, Potentially stable pattern, Gröbner basis.

AMS subject classifications. 15B35, 15A18, 15A24, 15A48, 05C05, $05 \mathrm{C} 50$.

1. Introduction and Preliminaries. In qualitative and combinatorial matrix theory, properties of a matrix based on combinatorial information, such as the signs of entries in the matrix, are studied. A matrix whose entries are from the set $\{+,-, 0\}$ is called a sign pattern matrix (or sign pattern, or pattern). We denote the set of all $n \times n$ sign pattern matrices by $Q_{n}$. For a real matrix $B, \operatorname{sgn}(B)$ is the sign pattern matrix obtained by replacing each positive (respectively, negative, zero) entry of $B$ by + (respectively,,- 0$)$. For a sign pattern matrix $A$, the sign pattern class of $A$ is defined by

$$
Q(A)=\{B: \operatorname{sgn}(B)=A\} .
$$

* Received by the editors February 23, 2009. Accepted for publication February 8, 2010. Handling Editor: Michael J. Tsatsomeros.

${ }^{\dagger}$ Department of Mathematics and Statistics, Georgia State University, Atlanta, GA 30302-4110, USA (marav@gsu.edu, fhall@gsu.edu, zli@gsu.edu, nmanzagol@gsu.edu).

$¥$ Department of Mathematics and Statistics, Texas Tech University, Lubbock, TX 79409-1042. 
A subpattern of a sign pattern $A$ is a sign pattern matrix obtained from $A$ by replacing a number (possibly none) of the + or - entries in $A$ with 0 . If $\hat{A}$ is a subpattern of $A$, we also say that $A$ is a superpattern of $\hat{A}$.

The sign pattern $I_{n} \in Q_{n}$ is the diagonal pattern of order $n$ with + diagonal entries. A sign pattern matrix $P$ is called a permutation pattern if exactly one entry in each row and column is equal to + , and all other entries are 0 . Two sign pattern matrices $A_{1}$ and $A_{2}$ are said to be permutationally similar if there is a permutation pattern $P$ such that $A_{2}=P^{T} A_{1} P$.

A signature (sign) pattern is a diagonal sign pattern all of whose diagonal entries are nonzero. Two sign pattern matrices $A_{1}$ and $A_{2}$ are said to be signature similar if there is a signature pattern $S$ such that $A_{2}=S A_{1} S$.

A sign pattern $A \in Q_{n}$ is said to be sign nonsingular (SNS) if every matrix $B \in Q(A)$ is nonsingular. It is well known that $A$ is sign nonsingular if and only if $\operatorname{det} A=+$ or $\operatorname{det} A=-$, that is, in the standard expansion of det $A$ into $n$ ! terms, there is at least one nonzero term, and all the nonzero terms have the same sign. This means that det $B$ is positive (or negative) for all $B \in Q(A)$.

A combinatorially symmetric sign pattern matrix is a square sign pattern $A$ where $a_{i j} \neq 0$ if and only if $a_{j i} \neq 0$. The graph $G(A)$ of a combinatorially symmetric $n \times n$ sign pattern matrix $A=\left[a_{i j}\right]$ is the graph with vertex set $\{1,2,3, \ldots, n\}$ where $\{i, j\}$ is an edge if and only if $a_{i j} \neq 0$. A tree sign pattern (tsp) is a combinatorially symmetric sign pattern matrix whose graph is a tree (possibly with loops).

The set of all eigenvalues (counting multiplicities) of a square matrix $B$ is denoted by $\sigma(B)$, and the inertia of matrix $B$ is the ordered triple

$$
i(B)=\left(i_{+}(B), i_{-}(B), i_{0}(B)\right),
$$

in which $i_{+}(B), i_{-}(B)$ and $i_{0}(B)$ are the numbers of elements of $\sigma(B)$ with positive, negative and zero real parts, respectively. The inertia set of a square sign pattern $A$ is the set of ordered triples $i(A)=\{i(B): B \in Q(A)\}$. An $n \times n$ sign pattern $A$ is said to be an inertially arbitrary pattern (IAP) if $(r, s, t) \in i(A)$ for every nonnegative integer triple $(r, s, t)$ with $r+s+t=n$.

An $n \times n$ matrix $B$ is stable if $i(B)=(0, n, 0)$. An $n \times n$ pattern $A$ is sign stable if $i(A)=\{(0, n, 0)\}$, and potentially stable (PS) if $(0, n, 0) \in i(A)$.

An $n \times n$ pattern $A$ is a spectrally arbitrary pattern (SAP) if, for any given real monic polynomial $r(x)$ of degree $n$, there is a matrix $B \in Q(A)$ with characteristic polynomial $r(x)$. That is, $A$ is an SAP if for any possible spectrum of a real matrix (namely, any set of $n$ complex numbers with nonreals occuring as conjugate pairs), there exists $B \in Q(A)$ with that spectrum. Clearly, if $A$ is an SAP, then $A$ is an IAP. 
However, it is known that the converse does not hold, see for example [2].

It is easily seen that the class of $n \times n$ SAPs (IAPs) is closed under negation, transposition, permutation similarity, and signature similarity. We say that two sign patterns are equivalent if one can be obtained from the other by using a sequence of such operations.

Every SAP $A$ must allow nilpotence (that is to say, $A$ is potentially nilpotent $(\mathrm{PN}))$. This follows by using $r(x)=x^{n}$. Also, by using $r(x)=(x+1)^{n}$, we see that every SAP $A$ is potentially stable. In fact, every IAP is potentially stable, since in particular, $(0, n, 0) \in i(A)$.

Of course, not every potentially nilpotent pattern is even an IAP. For instance, $\left[\begin{array}{cc}0 & + \\ 0 & 0\end{array}\right]$ is a potentially nilpotent pattern that is not an IAP. Also, $\left[\begin{array}{cc}- & + \\ 0 & -\end{array}\right]$ is an example of a potentially stable pattern that is not an IAP.

A sign pattern $A$ is a minimal inertially arbitrary pattern (MIAP) if $A$ is an IAP, but is not an IAP if one or more nonzero entries is replaced by zero. Analogously, $A$ is a minimal spectrally arbitrary pattern (MSAP) if $A$ is an SAP, but is not an SAP if one or more nonzero entries is replaced by zero.

There has been considerable interest recently in spectrally arbitrary sign patterns, which were introduced in [6]. In [1] it was established that any spectrally arbitrary sign pattern of order $n$ must have at least $2 n-1$ nonzero entries and conjectured that any spectrally arbitrary sign pattern of order $n$ must have at least $2 n$ nonzero entries. (This is known as the $2 n$-conjecture). In [1] and also in [2], the $3 \times 3$ spectrally arbitrary sign patterns were classified and demonstrated to have at least six nonzero entries.

Spectrally arbitrary tree sign patterns, especially those whose graphs (excluding loops) are paths, are considered in [6]. A method, based on the implicit function theorem, for proving that a sign pattern (and all superpatterns) is an SAP is developed there. A class of spectrally arbitrary patterns is constructed in [12] by using a Soules matrix. The implicit function theorem method is used in [1] to show that some Hessenberg sign patterns are minimal SAPs, the first such families for all orders to be presented. Other spectrally arbitrary sign pattern classes are constructed in [2] also by using the implicit function theorem method. In [4] the authors characterize all the $4 \times 4$ zero-nonzero patterns that are spectrally arbitrary. All potentially stable star sign patterns are characterized in [9]. The inertias of matrices having a symmetric star sign pattern are characterized in [16]. Potentially nilpotent star sign patterns are considered in [17], in which explicit characterizations are given for star sign patterns of orders two and three, and a recursive characterization for star sign patterns of general order $n$ is proved. In [14], it is shown that all potentially nilpotent entrywise 
nonzero sign patterns are spectrally arbitrary.

In this paper, tree sign patterns are investigated, with a special emphasis on $4 \times 4$ tridiagonal sign patterns. The set of spectrally arbitrary sign patterns is a subset of the set of potentially stable sign patterns, and for tree sign patterns of order 4 , the set of all potentially stable sign patterns is known (from [10] and [11] for paths and from [13] for stars). The main results are obtained by determining which of these potentially stable sign patterns are SAPs. All irreducible tridiagonal $4 \times 4$ SAPs are identified. Necessary and sufficient conditions for an irreducible $4 \times 4$ tridiagonal pattern to be an SAP are found. As a result, all $4 \times 4$ tree sign patterns that are SAPs are characterized. A new technique, an innovative application of Gröbner bases for demonstrating that a sign pattern is not potentially nilpotent, is introduced. Connections between the SAP (IAP) classes and the classes of potentially nilpotent and potentially stable patterns are explored. Some interesting open questions are also provided.

2. Tree Sign Patterns. For a sign pattern matrix whose undirected graph is a tree, the matrix is irreducible if and only if it is combinatorially symmetric , i.e., $a_{i j} \neq 0$ whenever $a_{j i} \neq 0$. Recall that we call such an irreducible sign pattern matrix a tree sign pattern (tsp). Suppose $A$ is an $n \times n$ tsp. Since $G(A)$ is a tree, $G(A)$ has $n-1$ edges. So, $A$ has $2(n-1)$ off-diagonal nonzero entries. In addition, if $A$ is an $\mathrm{SAP}$, then it has at least one positive and one negative diagonal entry. Hence, $A$ has at least $2 n$ nonzero entries.

It is easily proved that up to equivalence, the tsp

$$
T_{2}=\left[\begin{array}{ll}
- & + \\
- & +
\end{array}\right]
$$

is the only $2 \times 2 \mathrm{SAP}$, IAP, MSAP and MIAP. The following result is also easily shown.

Proposition 2.1. For $n=3$, if $A$ is a tsp, then $A$ is permutation similar to a tridiagonal pattern.

¿From [6], we have the following result.

Proposition 2.2. Up to equivalence,

$$
T_{3}=\left[\begin{array}{ccc}
- & + & 0 \\
- & 0 & + \\
0 & - & +
\end{array}\right], U=\left[\begin{array}{ccc}
- & + & 0 \\
- & + & + \\
0 & + & -
\end{array}\right], \tilde{T}_{3}=\left[\begin{array}{ccc}
- & + & 0 \\
- & + & + \\
0 & - & +
\end{array}\right]
$$

are the only $3 \times 3$ tsp SAPs.

Generalizing $T_{3}$, we have the following $n \times n$ antipodal sign pattern $T_{n}$. 
Theorem 2.3. For $2 \leq n \leq 16$,

$$
T_{n}=\left[\begin{array}{cccccc}
- & + & 0 & \cdots & \cdots & 0 \\
- & 0 & + & \ddots & & \vdots \\
0 & - & 0 & + & \ddots & \vdots \\
\vdots & \ddots & \ddots & \ddots & \ddots & 0 \\
\vdots & & \ddots & - & 0 & + \\
0 & \cdots & \cdots & 0 & - & +
\end{array}\right]
$$

is an $S A P$.

This follows from results in [6] and [7]. For $2 \leq n \leq 16, T_{n}$ is in fact an MSAP.

Up to equivalence, a $4 \times 4 \operatorname{tsp} A$ is a star pattern or a tridiagonal pattern. First, consider the $4 \times 4$ tsp SAPs with 8 nonzero entries. As indicated in [6], up to equivalence, we have just

$$
T_{4}=\left[\begin{array}{cccc}
- & + & 0 & 0 \\
- & 0 & + & 0 \\
0 & - & 0 & + \\
0 & 0 & - & +
\end{array}\right] \text { and } H=\left[\begin{array}{cccc}
- & + & 0 & 0 \\
+ & 0 & + & 0 \\
0 & - & 0 & + \\
0 & 0 & + & +
\end{array}\right]
$$

These patterns are actually MSAPs as they have the smallest possible number (8) of nonzero entries. We also note that the pattern $H$ is the last pattern given in Appendix $\mathrm{A}$ in [4].

What about the $4 \times 4$ tsp SAPs with more than 8 nonzero entries? For $n \times n$ star patterns we have the following result from [13].

Proposition 2.4. If $n \geq 2$ and $S_{n}$ is a star sign pattern, then the following are equivalent:

1. $S_{n}$ is equivalent to one of the patterns $Y_{n}, Z_{n p}, Z_{n p}^{+}$or $Z_{n p}^{-}$defined in [13] (for appropriate $p$ ).

2. $S_{n}$ is spectrally arbitrary.

3. $S_{n}$ is inertially arbitrary.

4. $S_{n}$ is potentially nilpotent and potentially stable.

We can then characterize the $4 \times 4$ SAP star sign patterns.

Corollary 2.5. $S_{4}$ is a star SAP if and only if $S_{4}$ is equivalent to one of the following patterns: 
$Y_{4}=\left[\begin{array}{cccc}+ & + & + & + \\ - & - & 0 & 0 \\ + & 0 & - & 0 \\ - & 0 & 0 & -\end{array}\right], Z_{41}=\left[\begin{array}{cccc}0 & + & + & + \\ - & - & 0 & 0 \\ + & 0 & + & 0 \\ - & 0 & 0 & +\end{array}\right], Z_{42}=\left[\begin{array}{cccc}0 & + & + & + \\ - & - & 0 & 0 \\ + & 0 & - & 0 \\ - & 0 & 0 & +\end{array}\right]$,

$Z_{41}^{+}=\left[\begin{array}{cccc}+ & + & + & + \\ - & - & 0 & 0 \\ + & 0 & + & 0 \\ - & 0 & 0 & +\end{array}\right], Z_{41}^{-}=\left[\begin{array}{cccc}- & + & + & + \\ - & - & 0 & 0 \\ + & 0 & + & 0 \\ - & 0 & 0 & +\end{array}\right], Z_{42}^{+}=\left[\begin{array}{cccc}+ & + & + & + \\ - & - & 0 & 0 \\ + & 0 & - & 0 \\ - & 0 & 0 & +\end{array}\right]$,

$Z_{42}^{-}=\left[\begin{array}{cccc}- & + & + & + \\ - & - & 0 & 0 \\ + & 0 & - & 0 \\ - & 0 & 0 & +\end{array}\right]$

We next analyze the $4 \times 4$ tridiagonal patterns.

3. $4 \times 4$ Tridiagonal Spectrally Arbitrary Sign Patterns. In the article [10], the authors identify the $4 \times 4$ potentially stable (PS) tridiagonal tsp's (although four additional patterns are given in [11]). Since an SAP is a PS pattern, to investigate the $4 \times 4$ tridiagonal SAPs we can consider these PS tridiagonal patterns.

The results of analyzing all of the potentially stable $4 \times 4$ tridiagonal tsp's given in [10] are contained in Proposition 3.1, Theorem 3.3, Remark 3.4, Theorem 3.6 and Theorem 3.7. Analogous results for the $4 \times 4$ potentially stable sign patterns that are not included in [10] are given after Theorem 3.7.

\section{Proposition 3.1.}

(a). The nine tridiagonal superpatterns of $T_{4}$ are SAPs.

(b). The nine tridiagonal superpatterns of $H$ are $S A P s$.

(c). The following patterns are not SAPs because they are SNS:

$$
\begin{aligned}
& {\left[\begin{array}{cccc}
0 & + & 0 & 0 \\
- & + & + & 0 \\
0 & - & 0 & + \\
0 & 0 & - & -
\end{array}\right],\left[\begin{array}{cccc}
0 & + & 0 & 0 \\
- & + & + & 0 \\
0 & - & - & + \\
0 & 0 & - & -
\end{array}\right],} \\
& {\left[\begin{array}{cccc}
+ & + & 0 & 0 \\
- & 0 & + & 0 \\
0 & - & - & + \\
0 & 0 & - & 0
\end{array}\right],\left[\begin{array}{cccc}
0 & + & 0 & 0 \\
- & + & + & 0 \\
0 & - & - & + \\
0 & 0 & - & 0
\end{array}\right] .}
\end{aligned}
$$


(d). The following patterns are not SAPs because they are not potentially nilpotent. More specifically, $A^{4}$ is not compatible (as defined in [8]) with the zero matrix.

$$
\left[\begin{array}{cccc}
0 & + & 0 & 0 \\
- & 0 & + & 0 \\
0 & - & + & + \\
0 & 0 & - & -
\end{array}\right],\left[\begin{array}{cccc}
0 & + & 0 & 0 \\
- & 0 & + & 0 \\
0 & - & - & + \\
0 & 0 & - & +
\end{array}\right],\left[\begin{array}{cccc}
0 & + & 0 & 0 \\
- & 0 & + & 0 \\
0 & + & - & + \\
0 & 0 & - & +
\end{array}\right] .
$$

(e). The 30 sign patterns in [10] which do not have a positive diagonal entry are not SAPs.

A very common and very well known method to show that a pattern is an SAP is the Nilpotent-Jacobian method (N-J method) which first appeared in [6]. The N-J method was restated in [1] as follows.

TheOREM 3.2. Let $A$ be an $n \times n$ sign pattern, and suppose that there exists some nilpotent $B \in Q(A)$ with at least $n$ nonzero entries $\left(b_{1}, \ldots, b_{n}\right)$. Let $X$ be a matrix obtained by replacing these entries in $B$ by variables $\left(x_{1}, \ldots, x_{n}\right)$. Let

$$
P_{B}(t)=t^{n}+c_{1} t^{n-1}+c_{2} t^{n-2}+\ldots+c_{n-1} t+c_{n} .
$$

If the Jacobian matrix $J$ of $c_{1}, c_{2}, \ldots, c_{n}$ with respect to $x_{1}, \ldots, x_{n}$ is nonsingular at $\left(x_{1}, \ldots, x_{n}\right)=\left(b_{1}, \ldots, b_{n}\right)$, then $A$ and every superpattern of $A$ is an SAP.

THEOREM 3.3. The following tridiagonal tsp's are SAPs:

$$
\begin{aligned}
& {\left[\begin{array}{cccc}
+ & + & 0 & 0 \\
- & - & + & 0 \\
0 & + & 0 & + \\
0 & 0 & - & -
\end{array}\right],\left[\begin{array}{cccc}
+ & + & 0 & 0 \\
- & 0 & + & 0 \\
0 & - & - & + \\
0 & 0 & - & +
\end{array}\right],\left[\begin{array}{cccc}
+ & + & 0 & 0 \\
- & 0 & + & 0 \\
0 & + & - & + \\
0 & 0 & - & +
\end{array}\right]} \\
& {\left[\begin{array}{cccc}
- & + & 0 & 0 \\
- & + & + & 0 \\
0 & - & - & + \\
0 & 0 & - & 0
\end{array}\right],\left[\begin{array}{cccc}
0 & + & 0 & 0 \\
- & - & + & 0 \\
0 & + & - & + \\
0 & 0 & - & +
\end{array}\right],\left[\begin{array}{cccc}
0 & + & 0 & 0 \\
+ & + & + & 0 \\
0 & - & - & + \\
0 & 0 & + & -
\end{array}\right]}
\end{aligned}
$$

Proof. The proof uses the N-J method. We give below the sign pattern, its nilpotent realization, and the matrix used for computing the Jacobian which turns out to be nonsingular.

$$
\left[\begin{array}{cccc}
+ & + & 0 & 0 \\
- & - & + & 0 \\
0 & + & 0 & + \\
0 & 0 & - & -
\end{array}\right],\left[\begin{array}{cccc}
15 & 625 / 3 & 0 & 0 \\
-1 & -14 & 1 / 3 & 0 \\
0 & 1 & 0 & 3 \\
0 & 0 & -1 & -1
\end{array}\right],\left[\begin{array}{cccc}
x_{1} & x_{2} & 0 & 0 \\
-1 & -14 & x_{3} & 0 \\
0 & 1 & 0 & x_{4} \\
0 & 0 & -1 & -1
\end{array}\right]
$$




$$
\begin{aligned}
& {\left[\begin{array}{cccc}
+ & + & 0 & 0 \\
- & 0 & + & 0 \\
0 & - & - & + \\
0 & 0 & - & +
\end{array}\right],\left[\begin{array}{cccc}
1 & 1 & 0 & 0 \\
-1 & 0 & 1 & 0 \\
0 & -1 & -2 & 1 \\
0 & 0 & -1 & 1
\end{array}\right],\left[\begin{array}{cccc}
x_{1} & x_{2} & 0 & 0 \\
-1 & 0 & x_{3} & 0 \\
0 & -1 & -2 & x_{4} \\
0 & 0 & -1 & 1
\end{array}\right]} \\
& {\left[\begin{array}{cccc}
+ & + & 0 & 0 \\
- & 0 & + & 0 \\
0 & + & - & + \\
0 & 0 & - & +
\end{array}\right],\left[\begin{array}{cccc}
3 & 1 & 0 & 0 \\
-1 & 0 & 55 & 0 \\
0 & 1 & \frac{-186}{55} & \frac{194481}{3025} \\
0 & 0 & -1 & \frac{21}{55}
\end{array}\right],\left[\begin{array}{cccc}
3 & x_{1} & 0 & 0 \\
-1 & 0 & x_{2} & 0 \\
0 & 1 & \frac{-186}{55} & x_{3} \\
0 & 0 & -1 & x_{4}
\end{array}\right] ;} \\
& {\left[\begin{array}{cccc}
- & + & 0 & 0 \\
- & + & + & 0 \\
0 & - & - & + \\
0 & 0 & - & 0
\end{array}\right],\left[\begin{array}{cccc}
-6 & 48 & 0 & 0 \\
-1 & 8 & 1 & 0 \\
0 & -1 & -2 & 3 \\
0 & 0 & -1 & 0
\end{array}\right],\left[\begin{array}{cccc}
-6 & x_{1} & 0 & 0 \\
-1 & 8 & x_{2} & 0 \\
0 & -1 & -x_{3} & x_{4} \\
0 & 0 & -1 & 0
\end{array}\right]} \\
& {\left[\begin{array}{cccc}
0 & + & 0 & 0 \\
- & - & + & 0 \\
0 & + & - & + \\
0 & 0 & - & +
\end{array}\right],\left[\begin{array}{cccc}
0 & 2 & 0 & 0 \\
-1 & -1 & 1 & 0 \\
0 & 1 & -1 & 2 \\
0 & 0 & -1 & 2
\end{array}\right],\left[\begin{array}{cccc}
0 & x_{1} & 0 & 0 \\
-1 & -1 & x_{2} & 0 \\
0 & 1 & -x_{3} & x_{4} \\
0 & 0 & -1 & 2
\end{array}\right]} \\
& {\left[\begin{array}{cccc}
0 & + & 0 & 0 \\
+ & + & + & 0 \\
0 & - & - & + \\
0 & 0 & + & -
\end{array}\right],\left[\begin{array}{cccc}
0 & 1 & 0 & 0 \\
4 & 2 & 1 & 0 \\
0 & -8 & -1 & 1 \\
0 & 0 & 1 & -1
\end{array}\right],\left[\begin{array}{cccc}
0 & x_{1} & 0 & 0 \\
4 & 2 & x_{2} & 0 \\
0 & -8 & -x_{3} & 1 \\
0 & 0 & 1 & x_{4}
\end{array}\right]}
\end{aligned}
$$

REMARK 3.4. There are three other sign patterns listed in [10] equivalent to three sign patterns in Theorem 3.3. Also listed in [10] are 11 proper tridiagonal superpatterns of sign patterns equivalent to sign patterns in Theorem 3.3.

The following discussion about Gröbner basis is based on [5]. Let $R$ be a commutative ring. Consider a subset $S$ of the multivariable polynomial $\operatorname{ring} R\left[x_{1}, x_{2}, \ldots, x_{n}\right]$. A zero or a solution of $S$ in $R$ (or some super-ring of $R$ ) is an $n$-tuple $\left(r_{1}, r_{2}, \ldots, r_{n}\right) \in$ $R^{n}$ with $P\left(r_{1}, r_{2}, \ldots, r_{n}\right)=0$ for every polynomial $P \in S$. It can be seen that an $n$-tuple $\left(r_{1}, r_{2}, \ldots, r_{n}\right) \in R^{n}$ is a solution of $S$ if and only if it is a solution of the ideal generated by $S$. Hilbert's Basis Theorem states that every ideal of a polynomial ring over a field is finitely generated. From this point on, $R$ is the field of real numbers. Let $M$ be a set of monomials in $R\left[x_{1}, x_{2}, \ldots, x_{n}\right]$. Suppose that a certain ordering of all the monomials is prescribed. Let init $(P)$, the initial monomial of a polynomial $P$, be the largest monomial appearing in $P$. Let $S$ be a subset of $R\left[x_{1}, x_{2}, \ldots, x_{n}\right]$ and $\operatorname{init}(S)$ the ideal generated by $\{\operatorname{init}(s): s \in S\}$. If $I$ is an ideal of $R\left[x_{1}, x_{2}, \ldots, x_{n}\right]$, then a finite subset $G=\left\{g_{1}, g_{2}, \ldots, g_{k}\right\}$ of $I$ is called a 
Gröbner basis of $I$ if $\left\{\operatorname{init}\left(g_{1}\right), \operatorname{init}\left(g_{2}\right), \ldots, \operatorname{init}\left(g_{k}\right)\right\}$ generates $\operatorname{init}(I)$. For any ideal $I$ of $R\left[x_{1}, x_{2}, \ldots, x_{n}\right]$ the following are true.

(1) I has a Gröbner basis relative to any monomial ordering.

(2) Every Gröbner basis $G$ of $I$ generates $I$.

It can be seen that for every subset $S$ of $R\left[x_{1}, x_{2}, \ldots, x_{n}\right]$ and a Gröbner basis $G$ of the ideal generated by $S$, the solution set of $S$ is the same as the solution set of $G$.

The following fact is somewhat well-known, but for completeness, we give a proof.

Lemma 3.5. An $n \times n$ complex matrix $B$ is nilpotent if and only if $\operatorname{tr}(B)=0$, $\operatorname{tr}\left(B^{2}\right)=0, \operatorname{tr}\left(B^{3}\right)=0, \ldots, \operatorname{tr}\left(B^{n}\right)=0$.

Proof. Let $\sigma(B)=\left\{\lambda_{1}, \lambda_{2}, \ldots, \lambda_{n}\right\}$. It is well known that the trace of a matrix is equal to the sum of its eigenvalues. Thus, the necessity is obvious.

We now prove sufficiency. If all the eigenvalues are zero, then $B$ is unitarily similar to a strictly upper triangular matrix, and hence $B$ is nilpotent. Now assume that $B$ has some nonzero eigenvalues. Let $\lambda_{1}, \lambda_{2}, \ldots, \lambda_{k}$ denote the distinct nonzero eigenvalues of $B$, with multiplicities $m_{1}, m_{2}, \ldots, m_{k}$. The system of equations $\operatorname{tr}\left(B^{s}\right)=0,1 \leq s \leq n$, can be written as:

$$
\begin{array}{cc}
m_{1} \lambda_{1}+m_{2} \lambda_{2}+\ldots+m_{k} \lambda_{k} & =0 \\
m_{1} \lambda_{1}^{2}+m_{2} \lambda_{2}^{2}+\ldots+m_{k} \lambda_{k}^{2} & =0 \\
\vdots & \\
m_{1} \lambda_{1}^{n}+m_{2} \lambda_{2}^{n}+\ldots+m_{k} \lambda_{k}^{n} & =0
\end{array}
$$

Regarding $m_{1}, m_{2}, \ldots, m_{k}$ as the variables, the coefficient matrix $F$ of the first $k$ equations in the above system is

$$
F=\left[\begin{array}{cccc}
\lambda_{1} & \lambda_{2} & \ldots & \lambda_{k} \\
\lambda_{1}^{2} & \lambda_{2}^{2} & \ldots & \lambda_{k}^{2} \\
\vdots & & & \vdots \\
\lambda_{1}^{k} & \lambda_{2}^{k} & \ldots & \lambda_{k}^{k}
\end{array}\right]=\left[\begin{array}{cccc}
1 & 1 & 1 & 1 \\
\lambda_{1} & \lambda_{2} & \ldots & \lambda_{k} \\
\lambda_{1}^{2} & \lambda_{2}^{2} & \ldots & \lambda_{k}^{2} \\
\vdots & & & \vdots \\
\lambda_{1}^{k-1} & \lambda_{2}^{k-1} & \ldots & \lambda_{k}^{k-1}
\end{array}\right] \operatorname{diag}\left(\lambda_{1}, \lambda_{2}, \ldots, \lambda_{k}\right),
$$

where the first factor is a Vandermonde matrix. Thus $F$ is nonsingular since the $k$ parameters $\lambda_{1}, \lambda_{2}, \ldots, \lambda_{k}$ are nonzero and distinct. Thus, the above system has only the trivial solution $m_{1}=m_{2}=\ldots=m_{k}=0$. But by assumption, $m_{1}, m_{2}, \ldots, m_{k}$ are the multiplicities of distinct eigenvalues, and so cannot be zeros. Hence each eigenvalue of $B$ must be zero. That is, $B$ is nilpotent.

Note that the above result remains valid when the last condition $\operatorname{tr}\left(B^{n}\right)=0$ is replaced by $\operatorname{det}(B)=0$. Indeed, $\operatorname{det}(B)=0$ implies that at least one of the 
eigenvalues of $B$ is zero. Hence, the above proof of sufficiency is still valid since $k \leq n-1$.

An SAP must be potentially nilpotent. Using Maple to compute a Gröbner basis of polynomials obtained using the necessary and sufficient conditions mentioned in the above remark for a matrix to be nilpotent, we can get the following results.

THEOREM 3.6. The following patterns with 9 nonzero entries are not potentially nilpotent so they are not SAP's:

$$
\begin{aligned}
& {\left[\begin{array}{cccc}
- & + & 0 & 0 \\
- & + & + & 0 \\
0 & - & - & + \\
0 & 0 & + & 0
\end{array}\right],\left[\begin{array}{cccc}
- & + & 0 & 0 \\
- & + & + & 0 \\
0 & + & - & + \\
0 & 0 & - & 0
\end{array}\right],\left[\begin{array}{cccc}
- & + & 0 & 0 \\
+ & + & + & 0 \\
0 & - & - & + \\
0 & 0 & + & 0
\end{array}\right],\left[\begin{array}{cccc}
0 & + & 0 & 0 \\
- & - & + & 0 \\
0 & - & - & + \\
0 & 0 & - & +
\end{array}\right],} \\
& {\left[\begin{array}{cccc}
+ & + & 0 & 0 \\
- & + & + & 0 \\
0 & - & - & + \\
0 & 0 & - & 0
\end{array}\right],\left[\begin{array}{cccc}
0 & + & 0 & 0 \\
- & + & + & 0 \\
0 & + & - & + \\
0 & 0 & - & +
\end{array}\right],\left[\begin{array}{cccc}
0 & + & 0 & 0 \\
- & + & + & 0 \\
0 & - & - & + \\
0 & 0 & + & -
\end{array}\right],\left[\begin{array}{cccc}
+ & + & 0 & 0 \\
- & - & + & 0 \\
0 & + & 0 & + \\
0 & 0 & + & -
\end{array}\right],} \\
& {\left[\begin{array}{cccc}
- & + & 0 & 0 \\
+ & 0 & + & 0 \\
0 & - & + & + \\
0 & 0 & - & -
\end{array}\right],\left[\begin{array}{cccc}
+ & + & 0 & 0 \\
- & 0 & + & 0 \\
0 & - & - & + \\
0 & 0 & + & -
\end{array}\right],\left[\begin{array}{cccc}
- & + & 0 & 0 \\
- & 0 & + & 0 \\
0 & - & + & + \\
0 & 0 & + & -
\end{array}\right]}
\end{aligned}
$$

Proof. Let $B \in Q(A)$, where the absolute values of the nonzero entries of $B$ are independent variables and $A$ is one of the above mentioned patterns. A Gröbner basis of the ideal generated by the polynomials $\operatorname{tr}(B), \operatorname{tr}\left(B^{2}\right), \operatorname{tr}\left(B^{3}\right)$, and $\operatorname{det}(B)$ contains a polynomial that does not have a positive zero. The following are the details of the above mentioned process. Let

$$
A=\left[\begin{array}{cccc}
- & + & 0 & 0 \\
- & + & + & 0 \\
0 & - & - & + \\
0 & 0 & + & 0
\end{array}\right] .
$$

By performing a suitable diagonal similarity if necessary, we may assume that a matrix $B \in Q(A)$ has the following form

$$
B=\left[\begin{array}{cccc}
-a & b & 0 & 0 \\
-1 & c & d & 0 \\
0 & -1 & -e & f \\
0 & 0 & 1 & 0
\end{array}\right],
$$


where the variables can take on any positive values.

The polynomials $\operatorname{tr}(B), \operatorname{tr}\left(B^{2}\right), \operatorname{tr}\left(B^{3}\right), \operatorname{det}(B)$ are as follows

$$
\begin{gathered}
-a+c-e \\
a^{2}-2 b+c^{2}-2 d+e^{2}+2 f \\
-a^{3}+3 a b-3 b c+c^{3}-3 c d+3 d e-e^{3}-3 e f \\
a c f-b f
\end{gathered}
$$

The Gröbner basis with total degree ordering $\operatorname{tdeg}(a, b, c, d, e, f)$ for the ideal generated by the above polynomials consists of the following polynomials, $a-c+e, c^{2}-c e+e^{2}-b-d+f,-d f+e^{2} f+f^{2}, f c d-d f e+e f^{2}, e^{3}+c d-2 d e+2 e f$, $b d f+c e f^{2}, b d e f+e f^{2} d-c f^{3}-e f^{3}, b d^{2} f+d^{2} f^{2}-2 d f^{3}+f^{4}$.

Note that the solutions of the system of equations $\operatorname{tr}(B)=0, \operatorname{tr}\left(B^{2}\right)=0, \operatorname{tr}\left(B^{3}\right)=0$, $\operatorname{det}(B)=0$ are the same as the zeros of the set of polynomials in a Gröbner basis. The equation $b d f+c e f^{2}=0$ cannot have a positive solution for $a, b, c, d, e, f$. Thus the system cannot have a solution where all the variables are positive.

The following are the matrix realizations of the remaining sign patterns in the theorem and the monomial orderings relative to which the Gröbner basis of the ideal generated by $\left\{\operatorname{tr}(B), \operatorname{tr}\left(B^{2}\right), \operatorname{tr}\left(B^{3}\right)\right.$, $\left.\operatorname{det}(B)\right\}$ contains a polynomial with no positive zero.

$$
\begin{aligned}
{\left[\begin{array}{cccc}
-a & b & 0 & 0 \\
-1 & c & d & 0 \\
0 & 1 & -e & f \\
0 & 0 & -1 & 0
\end{array}\right], } & , \operatorname{tdeg}(a, b, c, d, e, f) ;\left[\begin{array}{cccc}
-a & b & 0 & 0 \\
1 & c & d & 0 \\
0 & -1 & -e & f \\
0 & 0 & 1 & 0
\end{array}\right], \operatorname{tdeg}(f, b, c, d, e, a) ; \\
& {\left[\begin{array}{cccc}
0 & a & 0 & 0 \\
-1 & -b & c & 0 \\
0 & -1 & -d & e \\
0 & 0 & -1 & f
\end{array}\right], \operatorname{tdeg}(f, b, c, d, e, a) ; } \\
& {\left[\begin{array}{cccc}
a & b & 0 & 0 \\
-1 & c & d & 0 \\
0 & -1 & -e & f \\
0 & 0 & -1 & 0
\end{array}\right], \operatorname{tddeg}(f, b, c, d, e, a) ; } \\
& {\left[\begin{array}{cccc}
0 & a & 0 & 0 \\
-1 & b & c & 0 \\
0 & 1 & -d & e \\
0 & 0 & -1 & f
\end{array}\right], \operatorname{tddeg}(f, e, c, d, b, a) ; }
\end{aligned}
$$




$$
\begin{aligned}
& {\left[\begin{array}{cccc}
0 & a & 0 & 0 \\
-1 & b & c & 0 \\
0 & -1 & -d & e \\
0 & 0 & 1 & -f
\end{array}\right], \operatorname{tdeg}(f, b, c, d, e, a) ;} \\
& {\left[\begin{array}{cccc}
a & b & 0 & 0 \\
-1 & -c & d & 0 \\
0 & 1 & 0 & e \\
0 & 0 & 1 & -f
\end{array}\right], \operatorname{tdeg}(a, b, c, d, e, f) ;} \\
& {\left[\begin{array}{cccc}
-a & b & 0 & 0 \\
1 & 0 & c & 0 \\
0 & -1 & d & e \\
0 & 0 & -1 & -f
\end{array}\right], \operatorname{tdeg}(f, b, c, d, e, a) ;} \\
& {\left[\begin{array}{cccc}
a & b & 0 & 0 \\
-b & 0 & c & 0 \\
0 & -c & -d & 1 \\
0 & 0 & 1 & -f
\end{array}\right], \operatorname{lexdeg}([d],[a, b, c, f]) ;} \\
& {\left[\begin{array}{cccc}
-a & b & 0 & 0 \\
-b & 0 & c & 0 \\
0 & -c & d & 1 \\
0 & 0 & 1 & -f
\end{array}\right], \operatorname{lexdeg}([d, a, c],[b, f])}
\end{aligned}
$$

Note that the second and the sixth patterns are actually equivalent.

THEOREM 3.7. The following patterns with 10 nonzero entries are not potentially nilpotent so they are not SAP's

$$
\begin{gathered}
{\left[\begin{array}{llll}
+ & + & 0 & 0 \\
- & - & + & 0 \\
0 & + & - & + \\
0 & 0 & + & -
\end{array}\right],\left[\begin{array}{llll}
+ & + & 0 & 0 \\
- & - & + & 0 \\
0 & + & + & + \\
0 & 0 & + & -
\end{array}\right],\left[\begin{array}{cccc}
+ & + & 0 & 0 \\
- & + & + & 0 \\
0 & - & - & + \\
0 & 0 & + & -
\end{array}\right],} \\
{\left[\begin{array}{cccc}
- & + & 0 & 0 \\
- & - & + & 0 \\
0 & - & + & + \\
0 & 0 & + & -
\end{array}\right],\left[\begin{array}{cccc}
- & + & 0 & 0 \\
+ & - & + & 0 \\
0 & - & + & + \\
0 & 0 & - & -
\end{array}\right],\left[\begin{array}{cccc}
+ & + & 0 & 0 \\
- & - & + & 0 \\
0 & - & - & + \\
0 & 0 & + & -
\end{array}\right],}
\end{gathered}
$$




$$
\left[\begin{array}{cccc}
- & + & 0 & 0 \\
+ & + & + & 0 \\
0 & - & + & + \\
0 & 0 & - & -
\end{array}\right],\left[\begin{array}{cccc}
+ & + & 0 & 0 \\
+ & - & + & 0 \\
0 & - & + & + \\
0 & 0 & - & -
\end{array}\right],\left[\begin{array}{cccc}
- & + & 0 & 0 \\
+ & - & + & 0 \\
0 & + & + & + \\
0 & 0 & - & -
\end{array}\right]
$$

Proof. The following are the matrix realizations and the term orderings for the Gröbner basis computation for the sign patterns in the theorem. In each case, applying a suitable diagonal similarity and multiplying by a suitable scalar matrix, we can make the absolute values of symmetric entries to be the same and the absolute values of the $(3,4)$ and $(4,3)$ entries to be 1 .

$$
\begin{aligned}
& {\left[\begin{array}{cccc}
a & b & 0 & 0 \\
-b & -c & d & 0 \\
0 & d & -e & 1 \\
0 & 0 & 1 & -f
\end{array}\right], \operatorname{tdeg}(a, b, c, d, e, f) .} \\
& {\left[\begin{array}{cccc}
a & b & 0 & 0 \\
-b & -c & d & 0 \\
0 & d & e & 1 \\
0 & 0 & 1 & -f
\end{array}\right], \operatorname{lexdeg}([a],[b, c, d, e, f]) .} \\
& {\left[\begin{array}{cccc}
a & b & 0 & 0 \\
-b & c & d & 0 \\
0 & -d & -e & 1 \\
0 & 0 & 1 & -f
\end{array}\right], \operatorname{lexdeg}([e],[a, b, c, d, f]) .} \\
& {\left[\begin{array}{cccc}
-a & \\
-a & b & 0 & 0 \\
-b & -c & d & 0 \\
0 & -d & e & 1 \\
0 & 0 & 1 & -f
\end{array}\right], \operatorname{lexdeg}([d, e, c],[a, b, f]) .}
\end{aligned}
$$




$$
\begin{aligned}
& {\left[\begin{array}{cccc}
-a & b & 0 & 0 \\
b & c & d & 0 \\
0 & -d & e & 1 \\
0 & 0 & -1 & -f
\end{array}\right], \operatorname{lexdeg}([c, d, e],[a, b, f]) .} \\
& {\left[\begin{array}{cccc}
a & b & 0 & 0 \\
b & -c & d & 0 \\
0 & -d & e & 1 \\
0 & 0 & -1 & -f
\end{array}\right], \operatorname{lexdeg}([c, d, e],[a, b, f]) .} \\
& {\left[\begin{array}{cccc}
-a & b & 0 & 0 \\
b & -c & d & 0 \\
0 & d & e & 1 \\
0 & 0 & -1 & -f
\end{array}\right], \operatorname{lexdeg}([c, d, e],[a, b, f]) .}
\end{aligned}
$$

The authors of [11] point out that Pang [15] conducted a systematic search of the potentially stable tree sign patterns for dimensions less than five. Based on the work in [15], the authors of [11] identify four minimally potentially stable sign patterns represented by $A_{4,8}$ to $A_{4,11}$ that are not contained in [10]. The pattern $A_{4,11}$ is the SAP $H$ discussed before. We convert $A_{4,8}$ and $A_{4,9}$ into tridiagonal form (which we still call $A_{4,8}$ and $A_{4,9}$ ) using permutation similarities, and then examine each of the tridiagonal superpatterns of $A_{4,8}, A_{4,9}$ and $A_{4,10}$.

We first note that

$$
A_{4,8}=\left[\begin{array}{cccc}
0 & + & 0 & 0 \\
+ & - & + & 0 \\
0 & - & + & + \\
0 & 0 & + & 0
\end{array}\right]
$$

is SNS, and hence not PN (and so it is not an SAP).

Note that the following two superpatterns of $A_{4,8}$ are equivalent:

$$
\left[\begin{array}{cccc}
0 & + & 0 & 0 \\
+ & - & + & 0 \\
0 & - & + & + \\
0 & 0 & + & -
\end{array}\right],\left[\begin{array}{cccc}
+ & + & 0 & 0 \\
+ & - & + & 0 \\
0 & - & + & + \\
0 & 0 & + & 0
\end{array}\right]
$$

Using a suitable Gröbner basis, we can show that the first (and hence, the second) is not $\mathrm{PN}$ :

$$
\left[\begin{array}{cccc}
0 & + & 0 & 0 \\
+ & - & + & 0 \\
0 & - & + & + \\
0 & 0 & + & -
\end{array}\right],\left[\begin{array}{cccc}
0 & a & 0 & 0 \\
b & -c & d & 0 \\
0 & -e & f & g \\
0 & 0 & h & -k
\end{array}\right], \operatorname{lexdeg}([e, f, d, g, a],[h, c, k, b])
$$


The other six tridiagonal superpatterns of $A_{4,8}$ are found to be SAPs since each of these is equivalent to a superpattern of $H$ or of a sign pattern in Theorem 3.3.

Next, we find that each of the tridiagonal superpatterns of

$$
A_{4,9}=\left[\begin{array}{cccc}
+ & - & 0 & 0 \\
+ & - & + & 0 \\
0 & + & - & + \\
0 & 0 & + & 0
\end{array}\right]
$$

is not PN, by using Gröbner bases:

$$
\begin{aligned}
A_{4,9}= & {\left[\begin{array}{cccc}
+ & - & 0 & 0 \\
+ & - & + & 0 \\
0 & + & - & + \\
0 & 0 & + & 0
\end{array}\right],\left[\begin{array}{cccc}
a & -b & 0 & 0 \\
c & -d & e & 0 \\
0 & f & -g & h \\
0 & 0 & k & 0
\end{array}\right], \operatorname{tdeg}(a, c, d, b, e, f, g, h, k) ; } \\
& {\left[\begin{array}{cccc}
+ & - & 0 & 0 \\
+ & - & + & 0 \\
0 & + & - & + \\
0 & 0 & + & +
\end{array}\right],\left[\begin{array}{cccc}
a & -1 & 0 & 0 \\
b & -c & 1 & 0 \\
0 & d & -e & 1 \\
0 & 0 & f & 1
\end{array}\right], \operatorname{lexdeg}([a, b, c],[d, e, f]), }
\end{aligned}
$$

where the Gröbner basis contains a polynomial equal to $(e-1)^{2} f+2 f+d f+1$;

$$
\left[\begin{array}{cccc}
+ & - & 0 & 0 \\
+ & - & + & 0 \\
0 & + & - & + \\
0 & 0 & + & -
\end{array}\right] \text { is the first matrix in Theorem 3.6. }
$$

Finally, the $(4,3)$ entry of the 4 th power of

$$
A_{4,10}=\left[\begin{array}{cccc}
- & + & 0 & 0 \\
- & + & + & 0 \\
0 & + & 0 & + \\
0 & 0 & - & 0
\end{array}\right]
$$

is -, so that $A_{4,10}$ is not $\mathrm{PN}$.

The only other tridiagonal superpattern of $A_{4,10}$ that was found to be not PN (by using Gröbner bases) is the following:

$$
\left[\begin{array}{cccc}
- & + & 0 & 0 \\
- & + & + & 0 \\
0 & + & - & + \\
0 & 0 & - & 0
\end{array}\right],\left[\begin{array}{cccc}
-a & 1 & 0 & 0 \\
-b & c & 1 & 0 \\
0 & d & -e & 1 \\
0 & 0 & -f & 0
\end{array}\right], \operatorname{tdeg}(a, b, c, d, e, f)
$$


The other seven tridiagonal superpatterns of $A_{4,10}$ are found to be SAPs since each of these is equivalent to a superpattern of a sign pattern in Theorem 3.3.

¿From the above discussion, theorems, propositions and remarks on tridiagonal patterns, we obtain the following theorem which provides necessary and sufficient conditions for a $4 \times 4$ irreducible tridiagonal pattern to be an SAP.

TheOREM 3.8. A $4 \times 4$ irreducible tridiagonal sign pattern is an SAP if and only if it is potentially stable and potentially nilpotent.

In fact, we have identified the $4 \times 4$ minimal irreducible tridiagonal SAPs, which are specified in the following theorem.

TheOREM 3.9. A $4 \times 4$ irreducible tridiagonal sign pattern is an SAP if and only if it is a superpattern of a sign pattern equivalent to one of the following minimal irreducible tridiagonal SAPs:

$$
\begin{aligned}
& T_{4}=\left[\begin{array}{cccc}
- & + & 0 & 0 \\
- & 0 & + & 0 \\
0 & - & 0 & + \\
0 & 0 & - & +
\end{array}\right], H=\left[\begin{array}{cccc}
- & + & 0 & 0 \\
+ & 0 & + & 0 \\
0 & - & 0 & + \\
0 & 0 & + & +
\end{array}\right] \\
& {\left[\begin{array}{cccc}
+ & + & 0 & 0 \\
- & - & + & 0 \\
0 & + & 0 & + \\
0 & 0 & - & -
\end{array}\right],\left[\begin{array}{cccc}
+ & + & 0 & 0 \\
- & 0 & + & 0 \\
0 & - & - & + \\
0 & 0 & - & +
\end{array}\right],\left[\begin{array}{cccc}
+ & + & 0 & 0 \\
- & 0 & + & 0 \\
0 & + & - & + \\
0 & 0 & - & +
\end{array}\right],} \\
& {\left[\begin{array}{cccc}
- & + & 0 & 0 \\
- & + & + & 0 \\
0 & - & - & + \\
0 & 0 & - & 0
\end{array}\right],\left[\begin{array}{cccc}
0 & + & 0 & 0 \\
- & - & + & 0 \\
0 & + & - & + \\
0 & 0 & - & +
\end{array}\right],\left[\begin{array}{cccc}
0 & + & 0 & 0 \\
+ & + & + & 0 \\
0 & - & - & + \\
0 & 0 & + & -
\end{array}\right]}
\end{aligned}
$$

Proposition 2.4, Corollary 2.5, and Theorem 3.8 then give us the following culminating result.

TheOrem 3.10. A $4 \times 4$ tree sign pattern is an SAP if and only if it is potentially stable and potentially nilpotent.

In the interesting paper [4], the authors characterize all the $4 \times 4$ zero-nonzero patterns that are spectrally arbitrary. For each spectrally arbitrary zero-nonzero pattern, a signing of that zero-nonzero pattern that is spectrally arbitrary is provided. However, as the authors point out, not every signing of a spectrally arbitrary zerononzero pattern will result in a spectrally arbitrary sign pattern. What we have done in this section is to precisely characterize the $4 \times 4$ tsp SAP's. 
4. Some Open Questions. It is known that if $A$ is an irreducible $3 \times 3$ pattern with a positive and negative diagonal entry, then $A$ is an SAP if and only if $A$ is PN. How far can we extend this result? It is not true in general for irreducible $4 \times 4$ patterns, as shown in [1]. But, what about for the class of $4 \times 4$ tridiagonal sign patterns? In this case, is PN (together with positive and negative diagonal entries) equivalent to being SAP? (This is to say, can we drop the potential stability condition in Theorem 3.8?)

More generally, can we determine (up to equivalence) the irreducible $4 \times 4 \mathrm{PN}$ patterns with a positive and a negative diagonal entry?

Does Theorem 3.10 somehow extend to the $5 \times 5$ case?

For $\mathrm{PN}$ patterns, is a rational realization always possible? That is, if $A$ is $\mathrm{PN}$, does there always exist a rational $B \in Q(A)$ that is nilpotent?

In [3], the authors give (up to equivalence) the irreducible $4 \times 4$ MIAPs that are not an SAP, namely

$$
\left[\begin{array}{llll}
* & * & 0 & 0 \\
0 & 0 & * & * \\
& * & 0 & 0 \\
0 & 0 & * & *
\end{array}\right], \quad\left[\begin{array}{llll}
* & * & * & 0 \\
& * & * & 0 \\
0 & 0 & 0 & * \\
& * & 0 & 0
\end{array}\right], \quad\left[\begin{array}{llll}
* & * & * & 0 \\
* & * & 0 \\
0 & 0 & * \\
0 & 0 & 0
\end{array}\right]
$$

Can this result be extended to $5 \times 5$ patterns, or $n \times n$, in general?

Acknowledgment. The authors are very grateful to Professor Pauline van den Driessche of the University of Victoria for reading earlier versions of this paper and for many useful comments. The authors are also very grateful to the referee for a careful reading of the paper and many valuable suggestions.

\section{REFERENCES}

[1] T. Britz, J.J. McDonald, D.D. Olesky, and P. van den Driessche. Minimal spectrally arbitrary patterns. SIAM J. Matrix Anal. Appl., 26:257-271, 2004.

[2] M.S. Cavers, and K.N. Vander Meulen. Spectrally and inertially arbitrary sign patterns. Linear Algebra Appl., 394:53-72, 2005.

[3] M.S. Cavers and K.N. Vander Meulen. Inertially arbitrary nonzero patterns of order 4. Electron. J. Linear Algebra, 16:30-43, 2007.

[4] L. Corpuz and J.J. McDonald. Spectrally arbitrary zero-nonzero patterns of order 4. Linear Multilinear Algebra, 55:249-273, 2007.

[5] D. Cox, J. Little, and D. O'Shea. Using Algebraic Geometry. Graduate Texts in Mathematics, Vol. 185, Springer, New York, 1998.

[6] J.H. Drew, C.R. Johnson, D.D. Olesky, and P. van den Driessche. Spectrally arbitrary patterns. Linear Algebra Appl., 308:121-137, 2000. 
[7] L. Elsner, D.D. Olesky, and P. van den Driessche. Low rank perturbations and the spectrum of a tridiagonal sign pattern. Linear Algebra Appl., 374:219-230, 2003.

[8] C.A. Eschenbach and Z. Li. Potentially nilpotent sign pattern matrices. Linear Algebra Appl., 299:81-99, 1999.

[9] Y. Gao and J. Li. On the potential stability of star sign pattern matrices. Linear Algebra Appl., 327:61-68, 2001.

[10] C.R. Johnson and T.A. Summers. The potentially stable tree sign patterns for dimensions less than five. Linear Algebra Appl., 126:1-13, 1989.

[11] Q. Lin, D.D. Olesky, and P. van den Driessche. The distance of potentially stable sign patterns to the unstable matrices, SIAM J. Matrix Anal. Appl., 24:356-367, 2002.

[12] J.J. McDonald, D.D. Olesky, M. Tsatsomeros, and P. van den Driessche. On the spectra of striped sign patterns. Linear Multilinear Algebra, 51:39-48, 2003.

[13] G. MacGillivray, R.M. Tifenbach, and P. van den Driessche. Spectrally arbitrary star sign patterns. Linear Algebra Appl., 400:99-119, 2005.

[14] Rajesh Pereira. Nilpotent matrices and spectrally arbitrary sign patterns. Electron. J. Linear Algebra, 16:232-236, 2007.

[15] J.C. Pang. Potential stability of tree sign patterns of dimension less than five. Work term report, Department of Mathematics and Statistics, University of Victoria, BC, Canada, 1996.

[16] Y. Shao, L. Sun, and Y. Gao. Inertia sets of two classes of symmetric sign patterns. Linear Algebra Appl., 384:85-95, 2004.

[17] L. Yeh. Sign patterns that allow a nilpotent matrix. Bull. Austral. Math. Soc., 53:189-196, 1996. 